Pacific

Journal of

Mathematics

FINITE-VOLUME COMPLEX-HYPERBOLIC SURFACES, THEIR TOROIDAL COMPACTIFICATIONS, AND GEOMETRIC APPLICATIONS

LuCA FABRIZIO Di CERBo

Volume $255 \quad$ No. 2

February 2012 


\title{
FINITE-VOLUME COMPLEX-HYPERBOLIC SURFACES, THEIR TOROIDAL COMPACTIFICATIONS, AND GEOMETRIC APPLICATIONS
}

\author{
LuCA FABRIZIo Di CERBo
}

\begin{abstract}
We study the classification of smooth toroidal compactifications of nonuniform ball quotients in the sense of Kodaira and Enriques. Several results concerning the Riemannian and complex algebraic geometry of these spaces are given. In particular we show that there are compact complex surfaces which admit Riemannian metrics of nonpositive curvature, but which do not admit Kähler metrics of nonpositive curvature. An infinite class of such examples arise as smooth toroidal compactifications of ball quotients.
\end{abstract}

\section{Introduction}

Let $\tilde{M}$ be a symmetric space of noncompact type, and let $\operatorname{Iso}_{0}(\tilde{M})$ denote the connected component of the isometry group of $\tilde{M}$ containing the identity. Recall that $\operatorname{Iso}_{0}(\tilde{M})$ is a semisimple Lie group. A discrete subgroup $\Gamma \subset \operatorname{Iso}_{0}(\tilde{M})$ is a lattice in $\tilde{M}$ if $\tilde{M} / \Gamma$ is of finite volume. When $\Gamma$ is torsion free, then $\tilde{M} / \Gamma$ is a finite volume manifold or a locally symmetric space. A lattice $\Gamma$ is uniform (nonuniform) if $\tilde{M} / \Gamma$ is compact (noncompact).

The theory of compactifications of locally symmetric spaces or varieties has been extensively studied, see for example [Borel and Ji 2006]. In fact, locally symmetric varieties of noncompact type often occur as moduli spaces in algebraic geometry and number theory, see [Ash et al. 2010]. For technical reasons this beautiful theory is mainly developed for quotients of symmetric spaces or varieties by arithmetic subgroups. For arithmetic subgroups of semisimple Lie groups a nice reduction theory is available [Borel and Ji 2006]. Among many other things, the aforementioned theory can be used to deduce their finite generation, the existence of finitely many conjugacy classes of maximal parabolic subgroups, and the existence of neat subgroups of finite index.

The celebrated work of Margulis [1984] implies that lattices in any semisimple Lie group of real rank bigger or equal than two are arithmetic subgroups. This important theorem does not cover many interesting cases such as lattices in the

MSC2010: 14J29, 53C20, 53C55.

Keywords: manifolds with nonpositive curvature, toroidal compactifications. 
complex hyperbolic space $\mathbb{C} \mathscr{H}^{n}$, where nonarithmetic lattices are known to exist by the work of Mostow and Mostow-Deligne; see [Deligne and Mostow 1993] and the bibliography therein.

It is thus desirable to develop a theory of compactifications of locally symmetric varieties modeled on $\mathbb{C}^{\mathscr{H}^{n}}$ regardless of the arithmeticity of the defining torsion free lattices. A compactification of finite-volume complex-hyperbolic manifolds as a complex spaces with isolated normal singularities was obtained by Siu and Yau [1982]. This compactification may be regarded as a generalization of the Baily-Borel compactification defined for arithmetic lattices in $\mathbb{C}^{\mathscr{C}^{n}}$. A toroidal compactification for finite-volume complex-hyperbolic manifolds was described by Hummel and Schroeder [1996] in connection with cusps closing techniques arising from Riemannian geometry; see also [Mok 2009] and the classical reference [Ash et al. 2010] for what concerns the arithmetic case.

The constructions of both Siu-Yau and Hummel-Schroeder rely on the theory of nonpositively curved Riemannian manifolds. The key point here is that the structure theorems for finite-volume manifolds of negatively pinched curvature, or more generally for visibility manifolds [Eberlein 1996], can be used as a substitute of the reduction theory for arithmetic subgroups.

In this paper we study torsion-free nonuniform lattices in the complex hyperbolic plane $\mathbb{C}_{\mathscr{H}^{2}}$ and their toroidal compactifications. Let $\Gamma$ be a lattice as above and let $\overline{\mathbb{C} \mathscr{H}^{2} / \Gamma}$ denote its toroidal compactification. When $\overline{\mathbb{C} \mathscr{H}^{2} / \Gamma}$ is smooth, it is a compact Kähler surface [Hummel 1998]. It is then of interest to place these smooth Kähler surfaces in the framework of the Kodaira-Enriques classification of complex surfaces [Barth et al. 2004]. The main purpose of this paper is to prove the following:

Theorem A. Let $\Gamma$ be a nonuniform torsion-free lattice in $\mathbb{C}^{2}$. There exists a finite subset $\mathscr{F}^{\prime} \subset \Gamma$ of parabolic isometries for which the following holds: for any normal subgroup $\Gamma^{\prime} \triangleleft \Gamma$ with the property that $\mathscr{F}^{\prime} \cap \Gamma^{\prime}$ is empty, then $\overline{\mathbb{C} \mathscr{H}^{2} / \Gamma^{\prime}}$ is a surface of general type with ample canonical line bundle. Moreover, $\overline{\mathbb{C} \mathscr{H}^{2} / \Gamma^{\prime}}$ admits Riemannian metrics of nonpositive sectional curvature but it cannot support Kähler metrics of nonpositive sectional curvature.

An outline of the paper follows. Section 2 starts with a summary of the results from [Hummel and Schroeder 1996]. Such results are then combined with the Kodaira-Enriques classification to prove that when the lattice $\Gamma$ is sufficiently small then $\overline{\mathbb{C} \mathscr{H}^{2} / \Gamma}$ is a surface of general type with ample canonical bundle.

In Section 3 we present some examples of a surfaces of general type which do not admit any nonpositively curved Kähler metric, but whose underlying smooth manifolds admit Riemannian metrics of nonpositive curvature. Finally we prove Theorem A. 
In Section 4 we show how Theorem A, combined with the theory of semistable curves on algebraic surfaces [Sakai 1980], can be used to address the problem of the projective-algebraicity of minimal compactifications (Siu-Yau) of finite-volume complex-hyperbolic surfaces. Those results are summarized in Theorem B. The result obtained is effective.

The projective-algebraicity of minimal compactifications was proved in [Mok 2009] through $L^{2}$-estimates for the $\bar{\partial}$-operator. This analytical approach works in any dimension.

\section{Toroidal compactifications and the Kodaira-Enriques classification}

Let PU $(1,2)$ denote the connected component of Iso $\left(\mathbb{C} \mathscr{H}^{2}\right)$ containing the identity. Let $\Gamma$ be a nonuniform torsion-free lattice of holomorphic isometries of the complex hyperbolic plane $\mathbb{C}_{\mathcal{H}^{2}}$, that is, $\Gamma \leq \mathrm{PU}(1,2)$. Recall that the locally symmetric space $\mathbb{C} \mathscr{H}^{2} / \Gamma$ has finitely many cusp ends $A_{1}, \ldots, A_{n}$ which are in one to one correspondence with conjugacy classes of the maximal parabolic subgroups of $\Gamma$ [Eberlein 1980]. The set of all parabolic elements of $\Gamma$ can be written as a disjoint union of subsets $\Gamma_{x}$, where $\Gamma_{x}$ is the set of all parabolic elements in $\Gamma$ having $x$ as their unique fixed point. Here $x$ is a point in the natural point set compactification of $\mathbb{C} \mathcal{H}^{2}$ obtained by adjoining points at infinity corresponding to asymptotic geodesic rays. Thus, given a cusp $A_{i}$, let us consider the associated maximal parabolic subgroup $\Gamma_{x_{i}} \leq \Gamma$ and the horoball $\mathrm{HB}_{x_{i}}$ stabilized by $\Gamma_{x_{i}}$. We then have that $\mathrm{HB}_{x_{i}} / \Gamma_{x_{i}}$ is naturally identified with $A_{i}$.

Recall that after choosing an Iwasawa decomposition [Eberlein 1996] for PU(1, 2), we get a identification of $\partial \mathrm{HB}$ with the three-dimensional Heisenberg Lie group $N$. Moreover, $N$ comes equipped with a left invariant metric and then we may view $\Gamma_{x_{i}}$ as a lattice in $\operatorname{Iso}(N)$. The cusps $A_{1}, \ldots, A_{n}$ are then identified with $N / \Gamma_{x_{i}} \times[0, \infty)$, for $i=1, \ldots, n$.

The isometry group of $N$ is isomorphic to the semi-direct product $N \rtimes U(1)$. We say that a lattice in $\operatorname{Iso}(N)$ is rotation free if it is a lattice in $N$, that is, if it is a lattice of left translations. A parabolic isometry $\phi \in \Gamma$ is called unipotent if it acts as a translation on its invariant horospheres.

We now briefly summarize some of the results from [Hummel 1998; Hummel and Schroeder 1996].

Theorem 2.1 (Hummel-Scroeder). Let $\Gamma$ be a nonuniform torsion-free lattice in $\mathbb{C}^{2}$. There exists a finite subset $\mathscr{F} \subset \Gamma$ of parabolic isometries such that, for any normal subgroup $\Gamma^{\prime} \triangleleft \Gamma$ with the property that $\mathscr{F} \cap \Gamma^{\prime}$ is empty, $\overline{\mathbb{C} \mathscr{H}^{2} / \Gamma^{\prime}}$ is smooth and Kähler.

Furthermore, using a cusp closing technique arising from Riemannian geometry they were able to prove: 
Theorem 2.2 (Hummel-Schroeder). Let $\Gamma$ be a nonuniform torsion-free lattice in $\mathbb{C}^{2}{ }^{2}$. Then there exists a finite subset $\mathscr{F}^{\prime} \subset \Gamma$ of parabolic isometries containing $\mathscr{F}$ such that if $\Gamma^{\prime} \triangleleft \Gamma$ is a normal subgroup with the property that $\mathscr{F}^{\prime} \cap \Gamma^{\prime}$ is empty, then $\overline{\mathbb{C} \mathscr{H}^{2} / \Gamma^{\prime}}$ admits a Riemannian metric of nonpositive sectional curvature.

A few remarks about these results. A nonuniform torsion-free lattice in $\mathbb{C}^{2}{ }^{2}$ admits a smooth toroidal compactification if its parabolic isometries are all unipotent. In the arithmetic case this is achieved by choosing a neat subgroup of finite index [Ash et al. 2010]. It is also interesting to observe that we have plenty of normal subgroups satisfying the requirements of Theorems 2.1 and 2.2 , in fact $\mathrm{PU}(1,2)$ is linear and then residually finite by a fundamental result of Mal'tsev [1940]. Finally, it is interesting to notice that in general one expects the strict inclusion $\mathscr{F}^{\prime} \supset \mathscr{F}^{\prime}$ to hold. Explicit examples can be derived from the construction of Hirzebruch [1984].

For simplicity, a compactification as in Theorem 2.2 will be referred to as a toroidal Hummel-Schroeder compactification.

Proposition 2.3. Let $M$ be a finite-volume complex-hyperbolic surface which admits a toroidal Hummel-Schroeder compactification. Then the Euler number of $\bar{M}$ is strictly positive.

Proof. The idea for the proof goes back to an unpublished result of J. Milnor about the Euler number of closed four-dimensional Riemannian manifolds having sectional curvatures along perpendicular planes of the same sign; see [Chern 1955]. Let $(\bar{M}, g)$ be the Riemannian manifold obtained by closing the cusps of $M$ under the condition of nonpositive curvature [Hummel and Schroeder 1996]. Let $\Omega$ be its curvature matrix. We can always choose [Chern 1955] a orthonormal frame $\left\{e_{i}\right\}_{i=1}^{4}$ such that $R_{1231}=R_{1241}=R_{1232}=R_{1242}=R_{1332}=R_{1341}=0$. Hence

$$
\begin{aligned}
\operatorname{Pf}(\Omega) & =\Omega_{2}^{1} \wedge \Omega_{4}^{3}-\Omega_{3}^{1} \wedge \Omega_{4}^{2}+\Omega_{4}^{1} \wedge \Omega_{3}^{2} \\
& =\left(R_{1221} R_{3443}+R_{1243}^{2}+R_{1331} R_{2442}+R_{1342}^{2}+R_{1441} R_{2332}+R_{1234}^{2}\right) d \mu_{g},
\end{aligned}
$$

where $\operatorname{Pf}(\Omega)$ is the Pfaffian of the skew symmetric matrix $\Omega$. The statement is now a consequence of Chern-Weil theory.

We can now use the Kodaira-Enriques classification of closed smooth surfaces [Barth et al. 2004] to derive the following theorem. The proof is in the spirit of the theory of nonpositively curved spaces.

Theorem 2.4. Let $M$ be a finite-volume complex-hyperbolic surface which admits a toroidal Hummel-Schroeder compactification. Then $\bar{M}$ is a surface of general type without rational curves.

Proof. Since $\bar{M}$ admits a Riemannian metric of nonpositive sectional curvature, the Cartan-Hadamard theorem [Petersen 2006] implies that the universal cover of $\bar{M}$ is diffeomorphic to the four-dimensional euclidean space. Consequently, 
$\bar{M}$ is aspherical and then it cannot contain rational curves. Moreover, the second Betti number of $\bar{M}$ is even since by construction it admits a Kähler metric. By the Kodaira-Enriques classification [Barth et al. 2004] we conclude that the Kodaira dimension of $\bar{M}$ cannot be negative.

From Proposition 2.3, we know that the Euler number of $\bar{M}$ is strictly positive. The minimal complex surfaces with Kodaira dimension equal to zero and positive Euler number are simply connected or with finite fundamental group. Since $\pi_{1}(\bar{M})$ is infinite, the Kodaira dimension of $\bar{M}$ is bigger or equal than one.

The fundamental group of an elliptic surface with positive Euler number is completely understood in terms of the orbifold fundamental group of the base of the elliptic fibration. More precisely, denoting by $\pi: S \rightarrow C$ the elliptic fibration, if $S$ has no multiple fibers then $\pi$ induces an isomorphism $\pi_{1}(S) \simeq \pi_{1}(C)$. If we allow multiple fibers we have the isomorphism $\pi_{1}(S) \simeq \pi_{1}^{\mathrm{Orb}}(C)$. For these results we refer to [Friedman and Morgan 1994]. We now show that $\bar{M}$ cannot be an elliptic surface. When $S$ has multiple fibers, $\pi_{1}(S)$ always has torsion and then it cannot be the fundamental group of a nonpositively curved manifold. If we assume $\pi_{1}(\bar{M}) \simeq \pi_{1}(C)$, the fact that $\pi_{1}(\bar{M})$ grows exponentially [Avez 1970] forces the genus of the Riemann surface $C$ to be bigger or equal than two. Since all closed geodesics in a manifold of nonpositive curvature are essential in $\pi_{1}$, we have that the fundamental group of the flats introduced in the compactification injects into $\pi_{1}(\bar{M})$ and then by assumption into $\pi_{1}(C)$. By elementary hyperbolic geometry this would imply that $\mathbb{Z} \oplus \mathbb{Z}$ acts as a discrete subgroup of $\mathbb{R}$, which is clearly impossible.

Corollary 2.5. A toroidal Hummel-Schroeder compactification has ample canonical line bundle.

Proof. By Theorem 2.4 we know that $\bar{M}$ is a minimal surface of general type without rational curves. The corollary follows from Nakai's criterion for ampleness of divisors on surfaces [Barth et al. 2004]. More precisely, since for a minimal surface of general type the self-intersection of the canonical divisor is strictly positive [ibid.], it suffices to show that $K_{\bar{M}} \cdot E>0$ for any effective divisor $E$. Thus, let $E$ be an irreducible divisor and assume $K_{\bar{M}} \cdot E=0$. By the Hodge index theorem we must have $E \cdot E<0$. By the adjunction formula $E$ must be isomorphic to a smooth rational curve with self-intersection -2 .

In the arithmetic case, part of the results contained in Theorem 2.4 can be derived from a theorem of Tai, see [Ash et al. 2010]. Furthermore, similar results for the so-called Picard modular surfaces are obtained by Holzapfel [1980].

\section{Examples}

In this section we present examples of surfaces of general type which do not admit nonpositively curved Kähler metrics, but such that their underlying smooth 
manifolds do admit Riemannian metrics with nonpositive Riemannian curvature. In order to do this one needs to understand the restrictions imposed by the nonpositive curvature assumption on the holomorphic curvature tensor.

Thus, define

$$
p=2 \operatorname{Re} \xi \quad \text { and } \quad q=2 \operatorname{Re} \eta
$$

where

$$
\xi=\xi^{\alpha} \partial_{\alpha} \quad \text { and } \quad \eta=\eta^{\alpha} \partial_{\alpha} .
$$

In real coordinates we have

$$
R(p, q, q, p)=R_{h i j k} p^{h} q^{i} q^{j} p^{k}
$$

while in complex terms

$$
\begin{aligned}
& R(\xi+\bar{\xi}, \eta+\bar{\eta}, \eta+\bar{\eta}, \xi+\bar{\xi}) \\
& \quad=R(\xi, \bar{\eta}, \eta, \bar{\xi})+R(\xi, \bar{\eta}, \bar{\eta}, \xi)+R(\bar{\xi}, \eta, \eta, \bar{\xi})+R(\bar{\xi}, \eta, \bar{\eta}, \xi) .
\end{aligned}
$$

We then have

$$
\begin{aligned}
& R_{h i j k} p^{h} q^{i} q^{j} p^{k} \\
& \quad=R_{\alpha \bar{\beta} \gamma \bar{\delta}} \xi^{\alpha} \eta^{\bar{\beta}} \eta^{\gamma} \xi^{\bar{\delta}}+R_{\alpha \overline{\beta \gamma} \delta} \xi^{\alpha} \eta^{\bar{\beta}} \eta^{\bar{\gamma}} \xi^{\delta}+R_{\bar{\alpha} \beta \gamma \bar{\delta}} \xi^{\bar{\alpha}} \eta^{\beta} \eta^{\gamma} \xi^{\bar{\delta}}+R_{\bar{\alpha} \beta \bar{\gamma} \delta} \xi^{\bar{\alpha}} \eta^{\beta} \eta^{\bar{\gamma}} \xi^{\delta} \\
& =R_{\alpha \bar{\beta} \gamma \bar{\delta}} \xi^{\alpha} \eta^{\bar{\beta}} \eta^{\gamma} \xi^{\bar{\delta}}-R_{\alpha \bar{\beta} \gamma \bar{\delta}} \xi^{\alpha} \eta^{\bar{\beta}} \eta^{\bar{\delta}} \xi^{\gamma}-R_{\alpha \bar{\beta} \gamma \bar{\delta}} \xi^{\bar{\beta}} \eta^{\alpha} \eta^{\gamma} \xi^{\bar{\delta}}+R_{\alpha \bar{\beta} \gamma \bar{\delta}} \xi^{\bar{\beta}} \eta^{\alpha} \eta^{\bar{\delta}} \xi^{\gamma} \\
& =R_{\alpha \bar{\beta} \gamma \bar{\delta}}\left(\xi^{\alpha} \eta^{\bar{\beta}} \eta^{\gamma} \xi^{\bar{\delta}}-\xi^{\alpha} \eta^{\bar{\beta}} \eta^{\bar{\delta}} \xi^{\gamma}-\xi^{\bar{\beta}} \eta^{\alpha} \eta^{\gamma} \xi^{\bar{\delta}}+\xi^{\bar{\beta}} \eta^{\alpha} \eta^{\bar{\delta}} \xi^{\gamma}\right) \\
& =R_{\alpha \bar{\beta} \gamma \bar{\delta}}\left(\xi^{\alpha} \eta^{\bar{\beta}}-\eta^{\alpha} \xi^{\bar{\beta}}\right) \overline{\left(\xi^{\delta} \eta^{\bar{\gamma}}-\eta^{\delta} \xi^{\bar{\gamma}}\right)} .
\end{aligned}
$$

If we assume the Riemannian sectional curvature to be nonpositive we have

$$
R_{h i j k} p^{h} q^{i} q^{j} p^{k}=R_{\alpha \bar{\beta} \gamma \bar{\delta}}\left(\xi^{\alpha} \eta^{\bar{\beta}}-\eta^{\alpha} \xi^{\bar{\beta}}\right) \overline{\left(\xi^{\delta} \eta^{\bar{\gamma}}-\eta^{\delta} \xi^{\bar{\gamma}}\right)} \leq 0 .
$$

In complex dimension two, the right hand side of the above equality reduces (after some manipulations) to

$$
\begin{aligned}
R_{\alpha \bar{\beta} \gamma \bar{\delta}}\left(\xi^{\alpha} \eta^{\bar{\beta}}-\right. & \left.\eta^{\alpha} \xi^{\bar{\beta}}\right) \overline{\left(\xi^{\delta} \eta^{\bar{\gamma}}-\eta^{\delta} \xi^{\bar{\gamma}}\right)} \\
=R_{1 \overline{1}} 1 \overline{1} & \left|\xi^{1} \eta^{\overline{1}}-\eta^{1} \xi^{\overline{1}}\right|^{2}+4 \operatorname{Re}\left\{R_{1 \overline{1} 1 \overline{2}}\left(\xi^{1} \eta^{\overline{1}}-\eta^{1} \xi^{\overline{1}}\right) \overline{\left(\xi^{2} \eta^{\overline{1}}-\eta^{2} \xi^{\overline{1}}\right)}\right\} \\
& +2 R_{1 \overline{1} 2 \overline{2}}\left\{\left|\xi^{1} \eta^{\overline{2}}-\eta^{1} \xi^{\overline{2}}\right|^{2}+\operatorname{Re}\left(\xi^{1} \eta^{\overline{1}}-\eta^{1} \xi^{\overline{1}}\right) \overline{\left(\xi^{2} \eta^{\overline{2}}-\eta^{2} \xi^{\overline{2}}\right)}\right\} \\
& +2 \operatorname{Re}\left\{R_{1 \overline{2} 1 \overline{2}}\left(\xi^{1} \eta^{\overline{2}}-\eta^{1} \xi^{\overline{2}}\right) \overline{\left(\xi^{2} \eta^{\overline{1}}-\eta^{2} \xi^{\overline{1}}\right)}\right\} \\
& +4 \operatorname{Re}\left\{R_{2 \overline{2} 1 \overline{2}}\left(\xi^{2} \eta^{\overline{2}}-\eta^{2} \xi^{\overline{2}}\right) \overline{\left(\xi^{2} \eta^{\overline{1}}-\eta^{2} \xi^{\overline{1}}\right)}\right\}+R_{2 \overline{2} 2 \overline{2}}\left|\xi^{2} \eta^{\overline{2}}-\eta^{2} \xi^{\overline{2}}\right|^{2} .
\end{aligned}
$$

Following Mostow and Siu [1980], we choose the ansatz

$$
\xi^{1}=i a, \quad \xi^{2}=-i, \quad \eta^{1}=a, \quad \eta^{2}=1
$$


where $a$ is a real number. We get the inequality

$$
R_{1 \overline{1} 1 \overline{1}} 4 a^{4}-2 R_{1 \overline{1} 2 \overline{2}} 4 a^{2}+R_{2 \overline{2} 2 \overline{2}} 4 \leq 0 .
$$

Since nonpositive Riemannian sectional curvature implies nonpositive holomorphic sectional curvature, we conclude that

$$
\left(R_{1 \overline{1} 2 \overline{2}}\right)^{2} \leq R_{1 \overline{1} 1 \overline{1}} R_{2 \overline{2} 2 \overline{2}} .
$$

Theorem 3.1. A toroidal Hummel-Schroeder compactification does not admit any Kähler metric with nonpositive Riemannian sectional curvature.

Proof. Let us proceed by contradiction. Consider one of the elliptic divisors added in the compactification. By the properties of submanifolds of a Kähler manifold [Kobayashi and Nomizu 1969], we have that the holomorphic sectional curvature tangent to the elliptic divisor has to be zero. Let us denote such a holomorphic sectional curvature by $R_{1 \overline{1} 1 \overline{1}}$. By the inequality (1), we conclude that $R_{1 \overline{1} 2 \overline{2}}=0$. As a result, the Ricci curvature tangent to the elliptic divisor has to be zero. We conclude that

$$
K_{\bar{M}} \cdot \Sigma=\int_{\Sigma} c_{1}\left(K_{\bar{M}}\right)=0,
$$

which contradicts the ampleness of $K_{\bar{M}}$, see Corollary 2.5.

Combining Theorems 2.4 and 3.1 with Corollary 2.5, we have thus proved Theorem A.

\section{Projective-algebraicity of minimal compactifications}

Let $\bar{M}$ be a smooth toroidal compactification of a finite-volume complex-hyperbolic surface $M$ and let $\Sigma$ denote the compactifying divisor. The set $\Sigma$ is exceptional and it can be blow down. The resulting complex surface, with isolated normal singularities, is usually referred as the minimal compactification of $M$ [Siu and Yau 1982]. In this section we address the problem of the projective-algebraicity of minimal compactifications of finite-volume complex-hyperbolic surfaces. This is motivated by a beautiful example of Hironaka, see [Hartshorne 1977, p. 417], which shows that by contracting a smooth elliptic divisor on an algebraic surface one can obtain a nonprojective complex space. In the arithmetic case, the projectivealgebraicity of minimal compactifications of finite-volume complex-hyperbolic surfaces is known by the work of Baily and Borel, see [Borel and Ji 2006].

For completeness, we recall the theory of semistable curves on algebraic surfaces and logarithmic pluricanonical maps as developed by Sakai [1980].

Let $\bar{M}$ be a smooth projective surface. Let $\Sigma$ be a reduced divisor having simple normal crossings on $\bar{M}$. 
Definition 4.1. The pair $(\bar{M}, \Sigma)$ is called minimal if $\bar{M}$ does not contain an exceptional curve $E$ of the first kind such that $E \cdot \Sigma \leq 1$.

We consider the logarithmic canonical line bundle $\mathscr{L}=K_{\bar{M}}+\Sigma$ associated to $\Sigma$. Given any integer $k$, define $\bar{P}_{m}=\operatorname{dim} H^{0}(\bar{M}, \mathcal{O}(m \mathscr{L}))$. If $\bar{P}_{m}>0$, we define the $m$-th logarithmic canonical map $\Phi_{m \mathscr{L}}$ of the pair $(\bar{M}, \Sigma)$ by

$$
\Phi_{m \mathscr{L}}(x)=\left[s_{1}(x), \ldots, s_{N}(x)\right],
$$

for any $x \in \bar{M}$ and where $s_{1}, \ldots, s_{N}$ is a basis for the vector space $H^{0}(\bar{M}, \mathscr{O}(m \mathscr{L}))$. At this point one introduces the notion of logarithmic Kodaira dimension exactly as in the closed smooth case. We denote this numerical invariant by $\bar{k}(M)$ where $M=\bar{M} \backslash \Sigma$. We refer to [Iitaka 1982] for further details.

Definition 4.2. A curve $\Sigma$ is semistable if it has only normal crossings and each smooth rational component of $\Sigma$ intersects the other components of $\Sigma$ in more than one point.

We next give a numerical criterion for a minimal semistable pair $(\bar{M}, \Sigma)$ to be of log-general type.

Proposition 4.3 [Sakai 1980]. Given a minimal semistable pair $(\bar{M}, \Sigma)$ we have that $\bar{k}(M)=2$ if and only if $\mathscr{L}$ is numerically effective and $\mathscr{L}^{2}>0$.

In what follows, we denote by $\mathscr{E}$ the set of irreducible curves $E$ in $\bar{M}$ such that $\mathscr{L} \cdot E=0$.

Theorem 4.4 [Sakai 1980]. Let $(\bar{M}, \Sigma)$ be a minimal semistable pair of log-general type. The map $\Phi_{m \mathscr{L}}$ is then an embedding modulo $\mathscr{E}$ for any $m \geq 5$.

It is then necessary to characterize the irreducible divisors in $\mathscr{E}$. In particular, we need the following proposition.

Proposition 4.5. Let $(\bar{M}, \Sigma)$ be a minimal semistable pair with $\bar{k}(M)=2$. Let $E$ be an irreducible curve such that $\mathscr{L} \cdot E=0$. If $E$ is not contained in $\Sigma$ then $E \simeq \mathbb{C} P^{1}$ and $E \cdot E=-2$.

Proof. Under these assumptions we know that $\mathscr{L}^{2}>0$. By the Hodge index theorem

$$
\mathscr{L}^{2}>0, \quad \mathscr{L} \cdot E=0 \quad \Longrightarrow \quad E^{2}<0 .
$$

But now $\mathscr{L} \cdot E=0$ which implies

$$
K_{\bar{M}} \cdot E=-\Sigma \cdot E \leq 0 .
$$

We then have $K_{\bar{M}} \cdot E=0$ if and only if $E$ does not intersect $\Sigma$. In this case $p_{a}(E)=0$ and then $E \simeq \mathbb{C} P^{1}$ and $E^{2}=-2$. Assume now that $K_{\bar{M}} \cdot E<0$, then $K_{\bar{M}} \cdot E=E^{2}=-1$ and therefore $E$ is an exceptional curve of the first kind such that $E \cdot \Sigma=1$. This contradicts the minimality of the pair $(\bar{M}, \Sigma)$. 
We are now ready to prove the main results of this section. Let $\mathbb{C} \mathscr{H}^{2} / \Gamma$ be a finitevolume complex-hyperbolic surface that admits a smooth toroidal compactification as in Theorem 2.4. We then have that $\overline{\mathbb{C} \mathscr{H}^{2} / \Gamma}$ is a surface of general type with compactification divisor consisting of smooth disjoint elliptic curves.

Proposition 4.6. Let $\bar{M}$ be a minimal surface of general type. Let $\Sigma$ be a reduced divisor whose irreducible components consist of disjoint smooth elliptic curves. Then $(\bar{M}, \Sigma)$ is a minimal semistable pair with $\bar{k}(M)=2$.

Proof. Recall that the canonical divisor of any minimal complex surface of nonnegative Kodaira dimension is numerically effective [Barth et al. 2004]. It follows that the adjoint divisor $\mathscr{L}$ is numerically effective. An elliptic curve on a minimal surface of general type has negative self intersection. Moreover, for a minimal surface of general type it is known that the self-intersection of the canonical divisor is strictly positive [ibid.]. By the adjunction formula, we have $\mathscr{L}^{2}=K_{\bar{M}}^{2}-\Sigma^{2}>0$. By Proposition 4.3, we conclude that $\bar{k}(M)=2$.

Let $\mathbb{C} \mathscr{H}^{2} \backslash \Gamma_{1}$ be a finite-volume complex-hyperbolic surface which admits a smooth toroidal compactification $\bar{M}_{1}$. Let $\left(\bar{M}_{1}, \Sigma_{1}\right)$ be the associated minimal semistable pair. By Theorem A, we can find a normal subgroup $\Gamma_{2} \triangleleft \Gamma_{1}$ of finite index such that the toroidal compactification $\bar{M}_{2}$ of $\mathbb{C}^{2} / \Gamma_{2}$ is a minimal surface of general type with compactification divisor $\Sigma_{2}$. Since

$$
\pi: \mathbb{C H}^{2} / \Gamma_{2} \rightarrow \mathbb{C} \mathscr{H}^{2} / \Gamma_{1}
$$

is an unramified covering we conclude that $\bar{k}\left(M_{1}\right)=\bar{k}\left(M_{2}\right)$ [Iitaka 1982]. But by Proposition 4.6 we know that $\bar{k}\left(M_{2}\right)=2$, it follows that $\left(\bar{M}_{1}, \Sigma_{1}\right)$ is a minimal semistable pair of log-general type. Let us summarize this argument into a proposition.

Proposition 4.7. Let $(\bar{M}, \Sigma)$ be a smooth pair arising as the toroidal compactification of a finite-volume complex-hyperbolic surface. The pair $(\bar{M}, \Sigma)$ is minimal and log-general.

The following theorem is the main result of the present section.

Theorem B. Let $(\bar{M}, \Sigma)$ be a smooth pair arising as the toroidal compactification of a finite-volume complex-hyperbolic surface. Then the associated minimal compactification is projective algebraic.

Proof. By Proposition 4.7, the minimal pair $(\bar{M}, \Sigma)$ is log-general. By Theorem 4.4 we know that $\Phi_{m \mathscr{L}}$ is an embedding modulo $\mathscr{E}$ for any $m \geq 5$. We clearly have that $\Sigma$ is contained in $\mathscr{E}$. We claim that there are no other divisors in $\mathscr{E}$. Assume the contrary. By Proposition 4.5, any other curve in $\mathscr{E}$ must be a smooth rational divisor 
$E$ with self-intersection minus two. The adjunction formula gives $K_{\bar{M}} \cdot E=0$ which implies $\Sigma \cdot E=0$. This is clearly impossible. By Theorem 4.4 for $m \geq 5$, the map

$$
\Phi_{m \mathscr{L}}: \bar{M} \rightarrow \mathbb{C} P^{N-1}
$$

gives a realization of the minimal compactification as a projective-algebraic variety.

For an approach to the projective-algebraicity problem through $L^{2}$-estimates for the $\bar{\partial}$-operator we refer to [Mok 2009].

\section{Acknowledgments}

I would like to thank Professor Claude LeBrun for his constant support and for constructive comments on the paper. I also would like to thank Professor Klaus Hulek for some useful bibliographical suggestions and the referee for pertinent comments on the manuscript.

\section{References}

[Ash et al. 2010] A. Ash, D. Mumford, M. Rapoport, and Y.-S. Tai, Smooth compactifications of locally symmetric varieties, 2nd ed., Cambridge Mathematical Library 10, Cambridge University Press, 2010. MR 2010m:14067 Zbl 1209.14001

[Avez 1970] A. Avez, "Variétés riemanniennes sans points focaux", C. R. Acad. Sci. Paris Sér. A-B 270 (1970), A188-A191. MR 41 \#961 Zbl 0188.26402

[Barth et al. 2004] W. P. Barth, K. Hulek, C. A. M. Peters, and A. Van de Ven, Compact complex surfaces, 2nd ed., Ergebnisse der Math. 4, Springer, Berlin, 2004. MR 2004m:14070 Zbl 1036.14016

[Borel and Ji 2006] A. Borel and L. Ji, Compactifications of symmetric and locally symmetric spaces, Math. Theory Appl. 16, Birkhäuser, Boston, 2006. MR 2007d:22030 Zbl 1100.22001

[Chern 1955] S.-s. Chern, "On curvature and characteristic classes of a Riemann manifold", $A b h$. Math. Sem. Univ. Hamburg 20 (1955), 117-126. MR 17,783e Zbl 0066.17003

[Deligne and Mostow 1993] P. Deligne and G. D. Mostow, Commensurabilities among lattices in PU $(1, n)$, Annals of Mathematics Studies 132, Princeton University Press, 1993. MR 95a:22013 Zbl 0826.22011

[Eberlein 1980] P. Eberlein, "Lattices in spaces of nonpositive curvature", Ann. of Math. (2) 111:3 (1980), 435-476. MR 82m:53040 Zbl 0401.53015

[Eberlein 1996] P. B. Eberlein, Geometry of nonpositively curved manifolds, Chicago Lectures in Mathematics 7, University of Chicago Press, 1996. MR 98h:53002 Zbl 0883.53003

[Friedman and Morgan 1994] R. Friedman and J. W. Morgan, Smooth four-manifolds and complex surfaces, Ergebnisse der Math. 27, Springer, Berlin, 1994. MR 95m:57046 Zbl 0817.14017

[Hartshorne 1977] R. Hartshorne, Algebraic geometry, Graduate Texts in Mathematics 52, Springer, New York, 1977. MR 57 \#3116 Zbl 0367.14001

[Hirzebruch 1984] F. Hirzebruch, "Chern numbers of algebraic surfaces: an example", Math. Ann. 266:3 (1984), 351-356. MR 85j:14069 Zbl 0504.14030

[Holzapfel 1980] R.-P. Holzapfel, "A class of minimal surfaces in the unknown region of surface geography”, Math. Nachr. 98 (1980), 211-232. MR 83a:14031 Zbl 0474.14022 
[Hummel 1998] C. Hummel, "Rank one lattices whose parabolic isometries have no rotational part", Proc. Amer. Math. Soc. 126:8 (1998), 2453-2458. MR 98j:22014 Zbl 0898.22005

[Hummel and Schroeder 1996] C. Hummel and V. Schroeder, "Cusp closing in rank one symmetric spaces", Invent. Math. 123:2 (1996), 283-307. MR 97e:53098 Zbl 0860.53025

[Iitaka 1982] S. Iitaka, Algebraic geometry, Graduate Texts in Mathematics 76, Springer, New York, 1982. MR 84j:14001 Zbl 0491.14006

[Kobayashi and Nomizu 1969] S. Kobayashi and K. Nomizu, Foundations of differential geometry, vol. II, Wiley, New York, 1969. MR 97c:53001b Zbl 0175.48504

[Mal'tsev 1940] A. Mal'tsev, "On the faithful representation of infinite groups by matrices.", Am. Math. Soc. Transl. (2) 45 (1940), 1-18. Zbl 0158.02905

[Margulis 1984] G. A. Margulis, "Arithmeticity of the irreducible lattices in the semisimple groups of rank greater than 1", Invent. Math. 76:1 (1984), 93-120. MR 85j:22021 Zbl 0551.20028

[Mok 2009] N. Mok, "Projective-algebraicity of minimal compactifications of complex-hyperbolic space forms of finite volume", preprint, University of Hong Kong, 2009.

[Mostow and Siu 1980] G. D. Mostow and Y. T. Siu, "A compact Kähler surface of negative curvature not covered by the ball”, Annals of Mathematics. Second Series 112:2 (1980), 321-360. MR 82f:53075 Zbl 0453.53047

[Petersen 2006] P. Petersen, Riemannian geometry, 2nd ed., Graduate Texts in Mathematics 171, Springer, New York, 2006. MR 2007a:53001 Zbl 1220.53002

[Sakai 1980] F. Sakai, "Semistable curves on algebraic surfaces and logarithmic pluricanonical maps", Math. Ann. 254:2 (1980), 89-120. MR 82f:14031 Zbl 0431.14011

[Siu and Yau 1982] Y. T. Siu and S. T. Yau, "Compactification of negatively curved complete Kähler manifolds of finite volume", pp. 363-380 in Seminar on Differential Geometry, edited by S. T. Yau, Ann. of Math. Stud. 102, Princeton Univ. Press, 1982. MR 83g:32027 Zbl 0493.53049

Received April 1, 2011. Revised November 2, 2011.

LUCA FABRIZIO Di CERBo

Mathematics Department

DUKE UNIVERSITY

BOX 90320

DURHAM, NC 27708

UNITED STATES

luca@math.duke.edu 


\title{
PACIFIC JOURNAL OF MATHEMATICS
}

\author{
http://pacificmath.org \\ Founded in 1951 by \\ E. F. Beckenbach (1906-1982) and F. Wolf (1904-1989)
}

\section{EDITORS}

V. S. Varadarajan (Managing Editor)

Department of Mathematics

University of California

Los Angeles, CA 90095-1555

pacific@math.ucla.edu

Vyjayanthi Chari

Department of Mathematics

University of California

Riverside, CA 92521-0135

chari@math.ucr.edu

\section{Robert Finn}

Department of Mathematics Stanford University

Stanford, CA 94305-2125

finn@math.stanford.edu

Kefeng Liu

Department of Mathematics

University of California

Los Angeles, CA 90095-1555

liu@math.ucla.edu
Darren Long

Department of Mathematics

University of California

Santa Barbara, CA 93106-3080

long@math.ucsb.edu

Jiang-Hua Lu

Department of Mathematics

The University of Hong Kong

Pokfulam Rd., Hong Kong jhlu@maths.hku.hk

Alexander Merkurjev

Department of Mathematics

University of California

Los Angeles, CA 90095-1555

merkurev@math.ucla.edu
Sorin Popa

Department of Mathematics University of California

Los Angeles, CA 90095-1555 popa@math.ucla.edu

Jie Qing

Department of Mathematics

University of California

Santa Cruz, CA 95064

qing@cats.ucsc.edu

Jonathan Rogawski

Department of Mathematics

University of California

Los Angeles, CA 90095-1555

jonr@math.ucla.edu

\section{PRODUCTION}

pacific@math.berkeley.edu

\section{SUPPORTING INSTITUTIONS}

ACADEMIA SINICA, TAIPEI

CALIFORNIA INST. OF TECHNOLOGY INST. DE MATEMÁTICA PURA E APLICADA KEIO UNIVERSITY

MATH. SCIENCES RESEARCH INSTITUTE NEW MEXICO STATE UNIV.

OREGON STATE UNIV.

\author{
STANFORD UNIVERSITY \\ UNIV. OF BRITISH COLUMBIA \\ UNIV. OF CALIFORNIA, BERKELEY \\ UNIV. OF CALIFORNIA, DAVIS \\ UNIV. OF CALIFORNIA, LOS ANGELES \\ UNIV. OF CALIFORNIA, RIVERSIDE \\ UNIV. OF CALIFORNIA, SAN DIEGO \\ UNIV. OF CALIF., SANTA BARBARA
}

\author{
UNIV. OF CALIF., SANTA CRUZ \\ UNIV. OF MONTANA \\ UNIV. OF OREGON \\ UNIV. OF SOUTHERN CALIFORNIA \\ UNIV. OF UTAH \\ UNIV. OF WASHINGTON \\ WASHINGTON STATE UNIVERSITY
}

These supporting institutions contribute to the cost of publication of this Journal, but they are not owners or publishers and have no responsibility for its contents or policies.

See inside back cover or pacificmath.org for submission instructions.

The subscription price for 2012 is US \$420/year for the electronic version, and \$485/year for print and electronic.

Subscriptions, requests for back issues from the last three years and changes of subscribers address should be sent to Pacific Journal of Mathematics, P.O. Box 4163, Berkeley, CA 94704-0163, U.S.A. Prior back issues are obtainable from Periodicals Service Company, 11 Main Street, Germantown, NY 12526-5635. The Pacific Journal of Mathematics is indexed by Mathematical Reviews, Zentralblatt MATH, PASCAL CNRS Index, Referativnyi Zhurnal, Current Mathematical Publications and the Science Citation Index.

The Pacific Journal of Mathematics (ISSN 0030-8730) at the University of California, c/o Department of Mathematics, 969 Evans Hall, Berkeley, CA 94720-3840, is published monthly except July and August. Periodical rate postage paid at Berkeley, CA 94704, and additional mailing offices. POSTMASTER: send address changes to Pacific Journal of Mathematics, P.O. Box 4163, Berkeley, CA 94704-0163.

PJM peer review and production are managed by EditFLOW ${ }^{\mathrm{TM}}$ from Mathematical Sciences Publishers.

PUBLISHED BY PACIFIC JOURNAL OF MATHEMATICS

at the University of California, Berkeley 94720-3840

A NON-PROFIT CORPORATION

Typeset in LATEX

Copyright $(02012$ by Pacific Journal of Mathematics 


\section{PACIFIC JOURNAL OF MATHEMATICS}

Volume $255 \quad$ No. $2 \quad$ February 2012

On the local Langlands correspondences of DeBacker-Reeder and

Reeder for $\operatorname{GL}(\ell, F)$, where $\ell$ is prime

MOSHE ADRIAN

$R$-groups and parameters

281

DUBRAVKA BAN and DAVID GOLDBERG

Finite-volume complex-hyperbolic surfaces, their toroidal

305

compactifications, and geometric applications

LUCA FABRIZIO Di CERBO

Character analogues of Ramanujan-type integrals involving the Riemann 317 $\Xi$-function

ATUl DiXIT

Spectral theory for linear relations via linear operators

DANA GHEORGHE and FLORIAN-HORIA VASILESCU

Homogeneous links and the Seifert matrix

PEDRO M. GonZÁlez MANCHÓN

Quantum affine algebras, canonical bases, and $q$-deformation of arithmetical functions

HENRY H. KIM and KYU-HWAN LEE

Dirichlet-Ford domains and arithmetic reflection groups

GRANT S. LAKELAND

Formal equivalence of Poisson structures around Poisson submanifolds

IOAN MĂRCUT,

A regularity theorem for graphic spacelike mean curvature flows

BENJAMIN STUART THORPE

Analogues of level- $N$ Eisenstein series 